\title{
Quantum Gravity in Relativistic Alpha Field Theory (QG in RAFT)
}

\author{
Branko M. Novakovic
}

\begin{abstract}
Classical quantum gravity approach is presented in the frame work of the new Relativistic Alpha Field Theory (RAFT). It is very important to describe gravity at the Planck's scale and near compact astrophysical objects where quantum effects cannot be ignored. Meanwhile, General Relativity Theory (GRT) cannot be applied to the extremely strong gravitational field at the Planck's scale, because of the related singularity. Recently developed RAF theory extends the application of GRT to the extremely strong fields, including Planck's scale. This is the consequence of determination of field parameters by using condition that they should satisfy both relativistic Hamiltonian and field equations with energy momentum tensor. As the result, we obtain, among the others, the following prediction of RAF theory: there exists a minimal gravitational radius at $r=\left(G M / 2 c^{2}\right)$ that prevents singularity at $r=0$. The existence of the minimal gravitational radius opens a new avenue for quantization of a gravitational field. One of the possible approaches is presented in this paper.
\end{abstract}

Index Terms- Quantum gravity, Relativistic Alpha Field Theory (RAFT), Minimal gravitational radius, Extremely strong gravitational fields

\section{INTRODUCTION}

It is of the special interest to describe gravity at the Planck's scale and near compact astrophysical objects where quantum effects cannot be ignored [1-5]. The main difficulties in quantum gravity (QG) description appear when one attempts to apply the usual prescriptions of quantum field theory to the force of gravity via graviton bosons [6]. The problem is that the theory one gets in this way is not renormalizable and therefore cannot be used to make meaningful physical predictions. Therefore, theorists have taken up more radical approaches to the problem of QG. The most popular approaches are string theory [7-10] and loop quantum gravity [11-15]. The noncommutative theories of geometry and an overview of many current approaches to QG are presented in [16-22]. The quantum cosmology problems and some collections of philosophers' texts of QG and popularization works are illustrated in [23-32].

As it is well known, the Planck's scale is far smaller in distance than those currently accessible at high energy particle accelerators. Therefore, QG is a mainly theoretical enterprise. Meanwhile, in the field of phenomenological QG, we have studies of the possibility of experimental tests of QG [33-37]. Unfortunately, at present, there is no QG theory which is universally accepted and confirmed by experience. One of the major hopes of observing traces of QG phenomena is in the cosmological context. In that sense, we have detection of an electromagnetic counterpart (GRB 170817A)

Branko Novakovic, FSB - University of Zagreb, Luciceva 5, P.O.B. 509, 10000 Zagreb, Croatia to the gravitational-wave signal (GW170817) from the merger of two neutron stars [38-41]. This opens a completely new arena for testing theories of gravity.

Further, General Relativity Theory (GRT) [42-47] cannot be applied to the extremely strong gravitational field at the Planck's scale, because of the related singularity. Here we apply a new theory that is called Relativistic Alpha Field (RAF) theory [48-50]. It has been shown [51] that RAF theory extends the capability of the GRT for the application to the extremely strong fields, including the Planck's scale. This is the consequence of determination of field parameters by using condition that they should satisfy both generalized relativistic Hamiltonian and field equations, with energy momentum tensor and without cosmological constant $\Lambda$ $[48,52]$. As the result, we obtain the following predictions of RAF theory: a) no a singularity at the Schwarzschild radius [49], b) there exists a minimal gravitational radius at $r=$ $\left(G M / 2 c^{2}\right)$ that prevents singularity at $r=0$, i.e. the nature protects itself [49], c) the gravitational force becomes positive (repulsive) if $\left(\boldsymbol{G M} / \boldsymbol{r c}^{2}\right)>\mathbf{1}$, that could be a source of a dark energy [50,53], and d) unification of electrical and gravitational forces can be done in the standard four dimensions (4D) [50]. The existence of the minimal gravitational radius opens a new avenue for quantization of a gravitational field.

The presented predictions of RAF theory may look like too speculative items. But, it is not because the solution of the field parameters generates energy - momentum tensor (EMT) on the right side of the field equations. Thus, this tensor is not added by the hand, as it is suggested in GRT. Furthermore, this EMT obeys the following required properties: symmetry, non-negative energy density and zero trace of EMT. Without EMT, the solution of the field parameters in RAF theory is reduced to the Schwarzschild's solution for the vacuum, as we expected that should be. This vacuum solution cannot be applied to the extremely strong fields, because of the related singularity. In that sense, it has been discussed the possibility that the singularity and dark energy problems are the consequences of vacuum solution of the field equations [54].

This paper is organized as follows. In Sec. II, we show the theoretical proof of the existence of the minimal radius. The quantum gravity procedures are presented in Sec. III. Some possible influences of the presented quantization are considered in Sec. IV. Finally, the related conclusion and the reference list are presented in Sec. V. and Sec. VI, respectively.

\section{EXISTENCE OF THE Minimal GRAVITATIONAL RADIUS}

Many arguments indicate that the Planck length may appear as a minimal meaningful length. But, in RAF theory [48-50] it has been predicted that there exists a minimal 
radius in gravitational field equal to $\boldsymbol{r}_{\boldsymbol{m}}=\boldsymbol{G M} / \mathbf{2} \boldsymbol{c}^{2}$, that eliminates the singularity problem. As we can see the minimal radius is proportional to mass. Thus, the minimal length belongs to the minimal mass. Minimal radius of the Planck's mass is half of the Planck's length $\boldsymbol{r}_{\boldsymbol{p} \boldsymbol{m}}=\boldsymbol{L}_{\boldsymbol{p}} / \mathbf{2}$. This means that the Planck's length is the minimal length only for the Planck's mass. As an example, the minimal radius of the

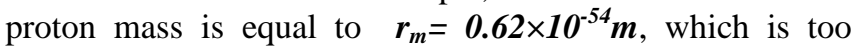
smaller than half of the Planck's length. Unfortunately, current technology is not yet capable of observing physical effects at scales that are so small. Therefore, we cannot confirm any of the current tentative QG theories, until the genuine quantum gravitational phenomena are directly or indirectly observed.

In order to theoretically prove the existence of the minimal radius in gravitational field, we started with the general line element $\mathrm{ds}^{2}$ in an alpha field, derived in the first part of RAF theory [48]. In the spherical polar coordinates this line element is presented by the nondiagonal form

$$
\begin{aligned}
d s^{2}= & -\alpha \alpha^{\prime} c^{2} d t^{2}-\kappa\left(\alpha-\alpha^{\prime}\right) c d t d r+d r^{2} \\
& +r^{2} d \theta^{2}+r^{2} \sin ^{2} \theta d \phi^{2} .
\end{aligned}
$$

Generally, field parameters $\alpha$ and $\alpha^{\prime}$ are scalar functions of the space-time coordinates, or normalized potential energy $\left(\boldsymbol{U} / \boldsymbol{m c}^{2}\right)$ in an alpha field [48]. Here $\boldsymbol{U}$ is potential energy, $\boldsymbol{m}$ is the related particle rest mass in that field, $\mathrm{c}$ is the speed of light in vacuum and parameter $\kappa= \pm 1$. Since, the line element (1) belongs to the well-known form of the Riemann's type line element, the contra-variant coordinates and components of the covariant metric tensor are given by the relations:

$$
\begin{aligned}
& d x^{0}=c d t, \quad d x^{1}=d r, \quad d x^{2}=d \theta, \quad d x^{3}=d \phi, \\
& g_{00}=-\alpha \alpha^{\prime}, \quad g_{01}=g_{10}=-\kappa\left(\alpha-\alpha^{\prime}\right) / 2, \\
& g_{11}=1, \quad g_{22}=r^{2}, \quad g_{33}=r^{2} \sin ^{2} \theta .
\end{aligned}
$$

Starting with the line element (1) we employ, for the convenient, the following substitutions:

$$
\eta=\alpha \alpha^{\prime}, \quad \beta=\kappa\left(\alpha^{\prime}-\alpha\right) / 2 .
$$

In that case the nondiagonal line element (1) is transformed into the new relation

$$
\begin{aligned}
d s^{2}= & -\eta c^{2} d t^{2}+2 \beta c d t d r \\
& +d r^{2}+r^{2} d \theta^{2}+r^{2} \sin ^{2} \theta d \phi^{2} .
\end{aligned}
$$

The determination of the parameters $\eta$ and $\beta$ in the gravitational field has been obtained by using condition that field parameters should satisfy both the generalized relativistic Hamiltonian [52] and field equations [48], with energy momentum tensor $T_{\mu \nu}$ and without cosmological constant

$$
R_{\mu \nu}-\frac{1}{2} g_{\mu \nu} R=k T_{\mu \nu}, k=\frac{8 \pi G}{c^{4}}, \mu, v=0,1,2,3 .
$$

Here $R_{\mu \nu}$ is Ricci tensor, $R$ is Ricci scalar, $G$ is the Newton's gravitational constant and $c$ is the speed of the light in a vacuum. Thus, the solutions of the parameters $\eta$ and $\beta$ for the static gravitational field have been presented in the first and second parts of RAF theory [48, 49]:

$$
\begin{aligned}
& \eta=1-\frac{2 G M}{r c^{2}}+\left(\frac{G M}{r c^{2}}\right)^{2}=\left(1-\frac{G M}{r c^{2}}\right)^{2}, \\
& \beta=\mp \kappa \sqrt{\frac{2 G M}{r c^{2}}\left(1-\frac{G M}{2 r c^{2}}\right)}, \quad \kappa= \pm 1 .
\end{aligned}
$$

Here $\boldsymbol{M}$ is gravitational mass of the spherically symmetric not rotating body. The quadratic term $\left(\boldsymbol{G M} / \boldsymbol{r c}^{2}\right)^{2}$ in (6) generates the energy - momentum tensor on the right side of the Einstein's field equations [48-50]:

$$
\begin{aligned}
& T_{\mu \eta}=\left(T_{00}, T_{01}, T_{10}, T_{11}, T_{22}, T_{33}\right), \\
& T_{\mu \eta}=\left(\eta,-\beta,-\beta,-1, r^{2}, r^{2} \sin ^{2} \theta\right) \frac{(G M)^{2}}{8 \pi G r^{4}} .
\end{aligned}
$$

It is easy to prove that the energy - momentum tensor (EMT) in (7) obeys the following required properties: symmetry, non-negative energy density and zero trace of EMT. In relatively weak field, as is in our solar system, or in vacuum, the quadratic term $\left(\boldsymbol{G M} / \boldsymbol{r c}^{2}\right)^{2}$ is too small and can be neglected. In that case EMT is vanishing and parameters in (6) are transformed into the solution corresponding to the Schwarzschild's solution for the vacuum:

$$
\eta=1-\frac{2 G M}{r c^{2}}, \quad \beta=\mp \kappa \sqrt{\frac{2 G M}{r c^{2}}}, \quad \kappa= \pm 1 .
$$

Including the parameters solutions from (6) into (4), the line element (4) can be presented in the final form:

$$
\begin{aligned}
d s^{2}= & -\left(1-\frac{G M}{r c^{2}}\right)^{2} c^{2} d t^{2} \\
& \mp 2 \kappa \sqrt{\frac{2 G M}{r c^{2}}\left(1-\frac{G M}{2 r c^{2}}\right)} c d t d r \\
& +d r^{2}+r^{2} d \theta^{2}+r^{2} \sin ^{2} \theta d \phi^{2} .
\end{aligned}
$$

Thus, it is very important to point out that RAF theory includes both the full form solution (6) and vacuum solution (6a) of the field equations (5).

From (6) and (8) we can theoretically prove the following predictions of RAF theory: a) no singularity at the Schwarzschild radius and $b$ ) there exist the minimal radius at the position $\boldsymbol{r}_{\boldsymbol{m}}=\left(\boldsymbol{G M} / \mathbf{2} \boldsymbol{c}^{2}\right)$ that prevents singularity at $\boldsymbol{r}=\mathbf{0}$, i.e. the nature protects itself:

$$
\begin{aligned}
& r_{s c h}=\frac{2 G M}{c^{2}}, \rightarrow \eta_{s c h}=\frac{1}{4}, \beta_{s c h}=\mp \kappa \sqrt{\frac{3}{4}}, \kappa= \pm 1, \\
& r_{m}=\frac{G M}{2 c^{2}}, \rightarrow \eta=1, \quad \beta=0, \quad r<r_{m} \rightarrow \beta=\beta_{i m}, \\
& r \rightarrow \infty, \eta \rightarrow 1, \beta \rightarrow 0, r=r_{c}=\frac{G M}{c^{2}}, \rightarrow \eta=0, \beta=1 .
\end{aligned}
$$

Following (9) we can see that at the Schwarzschild radius, $r_{\text {sch }}$, parameters $\eta$ and $\beta$ are regular. This proves the prediction a): no singularity at the Schwarzschild radius. 
Further, from (9), we also can see that at the minimal radius, $\boldsymbol{r}_{\boldsymbol{m}}=\boldsymbol{G} \boldsymbol{M} / \boldsymbol{2} \boldsymbol{c}^{2}$, parameters $\eta$ and $\beta$ are also regular and for $r<r_{m}$ parameter $\beta$ becomes imaginary number, $\beta=\beta_{\text {im }}$. This proves the prediction $b$ ): there exists a minimal radius at $\boldsymbol{r}_{\boldsymbol{m}}=\left(\boldsymbol{G M} / \mathbf{2} \boldsymbol{c}^{2}\right)$ that prevents singularity at $\boldsymbol{r}=\boldsymbol{0}$. It seems that the existence of the minimal radius tells us that the nature protect itself from the singularity. Thus, we can say that the metrics of the line element in (4-6) is regular for a gravitational field in the region $\boldsymbol{r}_{\boldsymbol{m}} \leq \boldsymbol{r} \leq \infty$. On that way the proofs of the propositions a) and b) are finished.

Now we shall consider the prediction c) of the RAF theory, given in $[49,50]$ : the gravitational force becomes positive (repulsive) if $\left(\boldsymbol{G M} / \boldsymbol{r} \boldsymbol{c}^{2}\right)>\mathbf{1}$, that could be a source of a dark energy. In order to theoretically prove this prediction, we can start with the solutions of the radial acceleration and radial force. For the time-invariant (or very slowly changed) gravitational field, the radial acceleration $\ddot{a}$ and force $F$ are presented by the relations [50]:

$$
\begin{aligned}
& \ddot{a}=-\frac{G M}{r^{2}}\left(1-\frac{G M}{r c^{2}}\right), \\
& F=m \ddot{a}=-\frac{m G M}{r^{2}}\left(1-\frac{G M}{r c^{2}}\right) .
\end{aligned}
$$

Here $m$ is a rest mass of a particle. Generally, the gravitational radial force in (10), describes the interactions in the strong fields. In the case of the relatively weak fields (as we have in our solar system) the radial force relation (10) is reduced to the well-known descriptions of the interactions in those fields:

$$
\ddot{a}=-\frac{G M}{r^{2}}, \quad F=m \ddot{a}=-\frac{m G M}{r^{2}} .
$$

Further, from (10) we can see that the gravitational force is negative (attractive) for $\left(\boldsymbol{G M} / \boldsymbol{r} \boldsymbol{c}^{2}\right)<\mathbf{l}$. This is the case in the relatively weak gravitational field, where the term $\left(\boldsymbol{G M} / \boldsymbol{r} \boldsymbol{c}^{2}\right)$ can be neglected. On the other hand, the gravitational force becomes positive (repulsive) if $\left(\boldsymbol{G M} / \boldsymbol{r} \boldsymbol{c}^{2}\right)>\mathbf{1}$. This is the case in the extremely strong gravitational field where the term $\left(\boldsymbol{G M} / \boldsymbol{r c}^{2}\right)$ has to be taken into account.

In our solar system the term $\left(\boldsymbol{G M} / \boldsymbol{r} \boldsymbol{c}^{2}\right)$ is too small and can be neglected. For an example, on the surface of our Sun the amount of this term is $\left(\boldsymbol{G M} / \boldsymbol{r c}^{2}\right) \approx 2.1193 \times 10^{-6}<<1$. On the surface of our planet Earth the related influence of the Sun to this term is $\left(\boldsymbol{G M} / \boldsymbol{r} \boldsymbol{c}^{2}\right) \approx 0.989 \times 10^{-8}<<1$. The gravitational influence of the planet Earth on its surface is $\left(\boldsymbol{G M} / \boldsymbol{r} \boldsymbol{c}^{2}\right) \approx$ $0.695 \times 10^{-9}<<1$. The presented amounts of the term $\left(\boldsymbol{G M} / \boldsymbol{r c}^{2}\right)$ in our solar system could be the answer to the question: why our experience is that gravitational force is only the negative (attractive) force?

If the term $\left(\boldsymbol{G M} / \boldsymbol{r} \boldsymbol{c}^{2}\right)=\mathbf{1}$, then the gravitational force is equal to zero. This is the very important situation that is occurring at the gravitational radius $\boldsymbol{r}_{\boldsymbol{c}}=\left(\boldsymbol{G M} / \boldsymbol{c}^{2}\right)$. This radius separates the regions of the attractive and repulsive forces in a gravitational field. At the minimal radius $\boldsymbol{r}_{\boldsymbol{m}}$ gravitational force is positive (repulsive): $\boldsymbol{F}_{\boldsymbol{m}}=\left(\mathbf{4} \boldsymbol{m} \boldsymbol{c}^{4}\right) / \boldsymbol{G M}$. Now, we can say that the nature protects itself from the singularity by producing the positive (repulsive) gravitational force at the minimal radius. Further, from (10) we can see that the gravitational force is positive (repulsive) in the region $\boldsymbol{r}_{\boldsymbol{m}} \leq \boldsymbol{r}<$ $r_{c}$, where $r_{m}=\left(G M / 2 c^{2}\right)$ and $r_{c}=\left(G M / c^{2}\right)$. At the Schwarzschild radius the gravitational force is negative (attractive) $\boldsymbol{F}_{\text {sch }}=-\left(\boldsymbol{m} \boldsymbol{c}^{4} / \mathbf{8 G M}\right)$ and belongs to the negative (attractive) set of gravitation forces in the region $\boldsymbol{r}_{\boldsymbol{c}}<\boldsymbol{r} \leq \infty$.

Thus, the previous consideration theoretically confirms the prediction c) of the RAF theory: the gravitational force becomes positive (repulsive) if $\left(\boldsymbol{G M} / \boldsymbol{r c}^{2}\right)>\mathbf{1}$ that could be a source of a dark energy [53].

\section{QUANTUM GRAVITY PROCEDURES}

Following (9) we can see that there are two exclusive points in a gravitational static field. The first one is at the minimal radius, $\boldsymbol{r}_{\boldsymbol{m}}=\left(\boldsymbol{G M} / \boldsymbol{2} \boldsymbol{c}^{2}\right)$, where the parameters $\eta=1$ and $\beta=0$. The second point is at the radius $\boldsymbol{r}_{\boldsymbol{c}}=\left(\boldsymbol{G M} / \boldsymbol{c}^{2}\right)$, where $\eta=0$ and $\beta=1$. We also can conclude that $\boldsymbol{r}_{\boldsymbol{c}}=\mathbf{2} \boldsymbol{r}_{\boldsymbol{m}}$. These two points are very important items and can be useful in derivation of the quantum gravity procedures. Namely, we can calculate the states of the velocities, accelerations (forces) and energies at the two exclusive points. Thus, it can be proved that at the point $\boldsymbol{r}_{\boldsymbol{m}}=\left(\boldsymbol{G M} / \mathbf{2} \boldsymbol{c}^{2}\right)$, radial velocity is equal to zero and radial acceleration (force) is positive and maximal. This means that a particle at this point will be repulsed to the bigger radius. Here we have repulsive (positive) gravitational force that also could be source of the so called dark energy [53]. On the other side, at the point $\boldsymbol{r}_{\boldsymbol{c}}=\left(\boldsymbol{G M} / \boldsymbol{c}^{2}\right)$, radial velocity is maximal and equal to the speed of the light in vacuum, and radial acceleration (force) is equal to zero [50]. If a gravitational radius $\boldsymbol{r}>\boldsymbol{r}_{\boldsymbol{c}}$, then radial velocity is decreasing and radial acceleration (force) becomes negative (attractive). Thus, the radius $\boldsymbol{r}_{\boldsymbol{c}}$ separates the region of the positive gravitational forces $\boldsymbol{r}_{\boldsymbol{m}} \leq \boldsymbol{r}<\boldsymbol{r}_{\boldsymbol{c}}$ from the region of the negative gravitational forces $\boldsymbol{r}_{\boldsymbol{c}}<\boldsymbol{r} \leq \infty$.

In order to make the quantization of a gravitation field we can start with determination of the potential energies of the particle at the minimal radius $\boldsymbol{r}_{\boldsymbol{m}}=\left(\boldsymbol{G M} / \mathbf{2} \boldsymbol{c}^{2}\right)$, and at the radius $r_{c}=\left(G M / c^{2}\right)$ :

$$
\begin{aligned}
& E_{1}=\frac{m G M}{r_{m}}=\frac{m G M}{G M / 2 c^{2}}=2 m c^{2}, \\
& E_{2}=\frac{m G M}{r_{c}}=\frac{m G M}{G M / c^{2}}=m c^{2} .
\end{aligned}
$$

Here $\mathrm{m}$ is a particle rest mass.

\section{A. Definition of graviton}

The transition from the energy state $E_{1}$ (at the minimal radius $r_{m}=r_{1}$ ) to the energy state $E_{2}$ (at the radius $r_{c}=r_{2}$ ) is resulted with the emission (radiation) of the graviton, as a quant of gravitational field, with the maximal energy:

$$
\begin{aligned}
& E_{1}-E_{2}=2 m c^{2}-m c^{2}=m c^{2}, \\
& h v_{1,2}=đ \omega_{1,2}=m c^{2} .
\end{aligned}
$$

Here $\boldsymbol{h}$ is the Planck's constant, $\boldsymbol{d}$ is the reduced Planck's constant, $\mathbf{v}_{\mathbf{1 , 2}}$ is the related gravitational wave frequency between energy states (levels) 1 and 2 and $\omega_{\mathbf{1 , 2}}$ is the related angular frequency. Thus, we can say that this quant of energy (graviton) is equal to the gravitational potential energy between the minimal radius $r_{\mathrm{m}}=r_{1}$ and the radius $r_{\mathrm{c}}=r_{2}$. In the opposite case, the transition from the energy state $E_{2}$ (at the 
radius $r_{c}=r_{2}$ ) to the energy state $E_{1}$ ( at the minimal radius $r_{m}=r_{1}$ ) is resulted with the absorption of the graviton, as a quant of gravitational field, with the same maximal energy. This is the physical concept of the quantization of a gravitational field.

\section{B. Quantization of gravitational radius, energy and frequency}

Quantization of the gravitational radius can be done by using the known values of the minimal radius $r_{m}$ and the radius $r_{c}$, given by (9):

$$
\begin{aligned}
& r_{1}=r_{m}=\frac{G M}{2 c^{2}}, \quad r_{2}=r_{c}=2 r_{m}=\frac{G M}{c^{2}}, \\
& r_{3}=3 r_{m}=3 \frac{G M}{2 c^{2}}, \ldots, \quad r_{n}=n r_{m}=n \frac{G M}{2 c^{2}} .
\end{aligned}
$$

Here $\boldsymbol{n}$ is quantization number of a gravitational field. Quantization of the gravitational potential energy follows from (11) and (13):

$$
\begin{aligned}
& E_{1}=\frac{m G M}{r_{1}}=2 m c^{2}, E_{2}=\frac{m G M}{r_{2}}=\frac{E_{1}}{2}=m c^{2}, \\
& E_{3}=\frac{m G M}{r_{3}}=\frac{2 m c^{2}}{3}=\frac{E_{1}}{3}, E_{n}=\frac{E_{1}}{n}, n=1,2, .
\end{aligned}
$$

In order to quantize the frequency $\boldsymbol{v}$ and the related angular frequency $\omega$ between two energy states (levels) in a gravitational field, we can employ (12) and (14):

$$
\begin{aligned}
& h v_{1,2}=\mathrm{d} \omega_{1,2}=E_{1}-E_{2}=E_{1}-\frac{E_{1}}{2}=\frac{E_{1}}{2}, \\
& h v_{2,3}=\mathrm{d} \omega_{2,3}=E_{2}-E_{3}=\frac{E_{1}}{2}-\frac{E_{1}}{3}=\frac{E_{1}}{6}, \\
& h v_{n-1, n}=đ \omega_{n-1, n}=\frac{E_{1}}{(n-1) n}, \quad n=2,3, . .
\end{aligned}
$$

Here $\boldsymbol{v}_{\mathbf{n}-\mathbf{1}, \mathbf{n}}$ is the gravitational wave frequency between energy states (levels) $(n-1)$ and $n$, where $n=2,3, .$. In that sense $\omega_{\mathbf{n}-\mathbf{1}, \mathbf{n}}$ is the related angular frequency. Thus, the quantization form of the frequency $\boldsymbol{v}$ and the related angular frequency $\omega$ between two energy states (levels) in a gravitational field are given by the relations:

$$
\begin{aligned}
& v_{n-1, n}=\frac{E_{1}}{(n-1) n h}=\frac{2 m c^{2}}{(n-1) n h}, \\
& \omega_{n-1, n}=\frac{2 m c^{2}}{(n-1) n d}, n=2,3, . .
\end{aligned}
$$

\section{Quantization of line element, energy momentum tensor, radial acceleration and force}

Now, we are ready to quantize the line element (8). In that sense, we started with parameters $\eta$ and $\beta$ from (6):

$$
\begin{aligned}
& \eta_{1}=\left(1-\frac{G M}{r_{1} c^{2}}\right)^{2}, \beta_{1}=\mp \kappa \sqrt{\frac{2 G M}{r_{1} c^{2}}\left(1-\frac{G M}{2 r_{1} c^{2}}\right)}, \\
& \kappa= \pm 1, r_{1}=r_{m}=\frac{G M}{2 c^{2}}, \quad r_{n}=n \cdot r_{m}=\frac{n G M}{2 c^{2}}, \\
& \eta_{n}=\left(1-\frac{2}{n}\right)^{2}, \beta_{n}=\mp \kappa \sqrt{\frac{4}{n}\left(1-\frac{1}{n}\right)}, n=1,2, . .
\end{aligned}
$$

Thus, the quantization form of the line element (8) is given by the relation:

$$
\begin{aligned}
d s_{n}^{2}= & -\left(1-\frac{2}{n}\right)^{2} c^{2} d t_{n}^{2} \mp 2 \kappa \sqrt{\frac{4}{n}\left(1-\frac{1}{n}\right)} c d t_{n} d r_{n} \\
& +d r_{n}^{2}+r_{n}^{2} d \theta_{n}^{2}+r_{n}^{2} \sin ^{2} \theta_{n} d \phi_{n}^{2}, n=1,2, .
\end{aligned}
$$

In order to quantize the energy-momentum tensor on the right side of the Einstein's field equations [48-50], we can employ the relation (7) and the previous quantization procedure:

$$
\begin{aligned}
& T_{n_{\mu \eta}}=\left(T_{n_{00}}, T_{n_{01}}, T_{n_{10}}, T_{n_{11}}, T_{n_{22}}, T_{n_{33}}\right), \\
& T_{n}=\left(\frac{2 c^{2}}{n}\right)^{4} \frac{1}{8 \pi G(G M)^{2}}, \quad n=1,2, ., \\
& T_{n_{00}}=\eta_{n} T_{n}, \quad T_{n_{01}}=T_{n_{10}}=-\beta_{n} T_{n}, \\
& T_{n_{11}}=-T_{n}, T_{n_{22}}=r_{n}{ }^{2} T_{n}, T_{n_{33}}=\sin ^{2} \theta_{n} T_{n_{22}} .
\end{aligned}
$$

Finally, the quantization forms of the radial acceleration $\ddot{a}$ and radial force F, given by (10), are presented by the following relations:

$$
\begin{aligned}
& \ddot{a}_{n}=-\frac{4 c^{4}}{n^{2} G M}\left(1-\frac{2}{n}\right), \quad F_{n}=m \ddot{a}_{n}, \\
& F_{n}=-\frac{4 c^{4}}{n^{2} G}\left(\frac{m}{M}\right)\left(1-\frac{2}{n}\right), n=1,2, ., \\
& m=M \rightarrow F_{n}=-\frac{4 c^{4}}{n^{2} G}\left(1-\frac{2}{n}\right) .
\end{aligned}
$$

From (20) we can see that the quantized gravitational radial force $F_{n}$ between two particles (bodies) with equal masses $(\boldsymbol{m}=\boldsymbol{M})$ is not function of the mass. At the minimal radius, $\boldsymbol{n}=\mathbf{1}$, the gravitational acceleration and force are positive (repulsive) and maximal. For the quantum number $\boldsymbol{n}=\mathbf{2}$, the gravitational acceleration and force are equal to zero. If the quantum number $\boldsymbol{n} \geq \mathbf{3}$, the gravitational acceleration and force are negative (attractive). Since our Universe is expanding at the acceleration rate, we can conclude that the present state of the Universe may be between the quantum states $\boldsymbol{n = 1}$ and $\boldsymbol{n = 2}$, but closer to the quantum state $\boldsymbol{n}=\mathbf{2}$. This could be an experimental test of RAF theory and of the presented quantum procedures of a gravitational field. At the same time, this means that between two quantum states there exists a transition process. At the Planck's scale this process is very, very short (looking from the macro time scale). On the other side, this process is very, very long at the macro time scale and should be taken into account. 


\section{Quantization of radial density}

Now, the quantization of the radial density $\rho=\boldsymbol{M} / \boldsymbol{r}$ is presented. This quantization is the function of the minimal radius:

$$
\begin{aligned}
& \rho_{1}=\rho_{M}=\frac{M}{r_{1}}=\frac{M}{r_{m}}=\frac{2 c^{2}}{G}, \\
& \rho_{2}=\frac{M}{r_{2}}=\frac{M}{r_{c}}=\frac{M}{2 r_{m}}=\frac{\rho_{M}}{2}=\frac{c^{2}}{G}, \\
& \rho_{n}=\frac{M}{r_{n}}=\frac{M}{n r_{m}}=\frac{\rho_{M}}{n}=\frac{2 c^{2}}{n G}, \quad n=1,2, . .
\end{aligned}
$$

Here $\rho_{\mathrm{M}}=2 \mathrm{c}^{2} / \mathrm{G}$ is the maximal radial density in a gravitational field. From (21) we can see that the radial density is not the explicit function of the mass. This means that all massive particles (bodies) have the same maximal radial density and the same radial density at each quantum number $\boldsymbol{\rho}_{\boldsymbol{n}}=\mathbf{2} \mathbf{c}^{\mathbf{2}} / \boldsymbol{n} \mathbf{G}, \boldsymbol{n}=\mathbf{1 , 2}, .$. This is very important discovery which predicts that radial density of any particle (body) cannot be greater than $\mathbf{2} \mathbf{c}^{\mathbf{2}} / \mathbf{G}$.

\section{INFLUENCES OF THE PRESENTED QUANTIZATION}

The presented quantization of a gravitational field could have very important influences to the regions like black holes, quantum field theory, high energy physics, Big Bang theory, dark energy, Universe acceleration, supernova explosion and cosmology. The presented quantization of gravitational field may also be useful in determination of the present Universe state and its maximal radius.

\section{CONCLUSION}

Recently developed Relativistic Alpha Field (RAF) theory [48-50] extends the capability of GRT for applications to the extremely strong fields, including Planck's scale [51]. One of the important predictions of RAF theory is the existence of the minimal gravitational radius equal to $\boldsymbol{r}_{m}=\left(\boldsymbol{G M} / 2 \boldsymbol{c}^{2}\right)$. This prediction eliminates the singularity problem in a strong gravitational field and opens a new avenue for the quantization of a gravitational field. One of the possible approaches is presented in this paper.

The quantization of the radial density shows that all massive particles (bodies) have the same maximal radial density $\rho_{\mathrm{M}}=2 \mathrm{c}^{2} / \mathrm{G}$, and the same radial density at each quantum number $\rho_{n}=2 \mathrm{c}^{2} / n \mathrm{G}$. Thus, the same maximal radial density have particles like electron and proton, as well as bodies like planet Earth, Sun and Universe. This is very important discovery which predicts that radial density of any particle (body) cannot be greater than $2 \mathrm{c}^{2} / \mathrm{G}$.

The presented predictions of RAF theory may look like too speculative items. But, it is not. This is just the consequence of determination of field parameters by using condition that they should satisfy both generalized relativistic Hamiltonian and field equations with energy momentum tensor on the right side of them.

\section{ACKNOWLEDGMENTS}

The author wishes to thank to the anonymous reviewers for a variety of helpful comments and suggestions. This work is supported by grants (120-1201842-3048) from the National Scientific Foundation of Republic of Croatia.

\section{REFERENCES}

[1] Rovelli C 2018 Quantum gravity (Scholarpedia www.scholarpedia.org) [2] Kiefer C 2012 Quantum gravity, (Oxford: Oxford University Press)

[3] Griffiths D J and David J 2004 Introduction to Quantum Mechanics (Pearson Prentice Hall, OCLC 803860989)

[4] Wald R M 1984 General Relativity (Chicago: University of Chicago Press $p 382$ OCLC $\underline{471881415}$ )

[5] Wald R M 1994 Quantum Field Theory in Curved Spacetime and Black Hole Thermodynamics (Chicago: University of Chicago Pres ISBN 0-226-87027-8)

[6] Zee A 2010 Quantum Field Theory in a Nutshell (Princeton: Princeton University Press $p 172$ OCLC 659549695)

[7] Green M, Schwarz J and Witten E 1987 Superstring Theory (Cambridge: Cambridge University Press)

[8] Polchinski J 1990 String Theory (Cambridge: Cambridge University Press)

[9] Blau M and Theisen S 2009 Gen. Relativ. Gravit. 41743

[10]Blumenhagen R, Lüst D and Theisen S 2013 Basic Concepts of String Theory (Berlin: Springer)

[11]Rovelli C 2004 Quantum Gravity (Cambridge: Cambridge University Press)

[12]Thiemann T 2007 Modern Canonical Quantum General Relativity (Cambridge: Cambridge University Press)

[13]Baez J C and Muniain J P 1994 Gauge Fields, Knots, and Gravity (World Scientific Publishing Company)

[14] Ashtekar A and Singh P 2011 Class. Quantum Grav. 28, 213001

[15]Rovelli C 2013 Covariant loop gravity (Quantum Gravity and Quantum Cosmology, pp 57-66 Lecture Notes in Physics 863 Berlin: Springer).

[16]Connes A 1994 Noncommutative Geometry (Sand Diego: Academic Press)

[17]Bastos C, Bertolami O, Costa Dias N and Nuno Prata J 2008 Phys. Rev. D 78, 023516

[18]Ashtekar A and Stachel J 1991 Conceptual Problems of Quantum Gravity (Boston-Basel-Berlin: Birkhauser)

[19]Oriti D 2008 Approaches to Quantum Gravity: Towards a New Understanding of Space, Time and Matter (Cambridge UP, Cambridge UK)

[20]Hamber H W 2009 Quantum gravitation - The Feynman path integral approach (Berlin: Springer)

[21]Pinto-Neto N, Falciano F T, Pereira R and Santini E S 2012 Phys. Rev. D 86063504

[22] Haug E G 2016 Int. J. Astron. Astrophys. 6, 206

[23]Penrose R 2009 J. Phys. Conf. Ser. 174012001

[24]Kamenshchik A Y, Kiefer C and Sandhöfer B 2007 Phys. Rev. D 76 064032

[25]Bojowald M 2012 Class. Quantum Grav. 29, 213001

[26]Callender C and Huggett N 2001 Physics Meets Philosophy at the Planck Scale: Contemporary Theories in Quantum Gravity (Cambridge UP, Cambridge UK)

[27]Rickles D, French S and Saatsi J 2007 The Structural Foundations of Quantum Gravity (Oxford: Oxford University Press)

[28]Smolin L 2000 Three Roads to Quantum Gravity (Oxford: Oxford University Press)

[29]Penrose R 2005 The road to reality (Oxford: Oxford University Press)

[30]Penrose $R 2007$ The road to reality: a complete guide to the laws of the universe (Vintage p 1017 OCLC 716437154 )

[31]Green B 1999 The Elegant Universe (New York: Vintage)

[32]Rovelli C 2004 What is Time? What is Space? (Rome: Di Renzo editore)

[33]Strominger A 2009 Nucl. Phys. Proc. Suppl. 192-193, 119-25

[34]Berti E at al 2015 Testing general relativity with present and future astrophysical observations Clas. Quantum Grav. 32243001 IOPscience (http://iopscience.iop.org/0264-0381/32/24/243001)

[35]Carlisle C 2014 First Direct Evidence of Big Bang Inflation (SkyandTelescope.com Retrieved March 18)

[36]Marongwel S 2017 Probing quantum gravity through strong gravitational lensing (DOI: 10.13140/RG.2.2.23029.88806)

[37]Dey S, Bhat A, Momeni D, Faizal M, Ali A F, Dey T K and Rehman A $2017 \quad$ Nuclear Physics B 10.1016/i.nuclphysb.2017.09.024 arXiv:1710.03920 [quant-ph] 
[38]Baker T, Bellini E, Ferreira P G, Lagos M, Noller J, and Sawicki I 2017 Phys. Rev. Lett. 119251301

[39]Creminelli P and Vernizzi F 2017 Phys. Rev. Lett. 119251302

[40]Sakstein J and Jain B 2017 Phys. Rev. Lett. 119251303

[41]Ezquiaga J M and Zumalacárregui M 2017 Phys. Rev. Lett. 119251304

[42]Einstein A 1916 Ann. Phys. 49 769-822

[43]Einstein A 1995 The Meaning of Relativity (Princeton: Princeton Univ. Press)

[44]Sean C 2003 Spacetime and Geometry: An Introduction to General Relativity (Amazon.com Books.htm, Hardcover)

[45]Weinberg S 1972 Gravitation and Cosmology: Principles and Application of the General Theory of Relativity (Gebundene Ausgabe, RelEspWeinberg.pdf.)

[46]S.W. Hawking S W and Ellis G F R 1973 The Large Scale Structure of Space-Time (Cambridge: Cambridge Univ. Press)

[47]Blau M 2014 Lecture Notes on General Relativity (Bern: A. Einstein Center for Fundamental Physics, Univ. Bern)

[48] Novakovic B M 2015 Int. J. New Techn. Res. 15 ID-IJNTR01050015 www.ijntr.org.

[49] Novakovic B M 2015 Int. J. New Techn. Res. 15 ID-IJNTR01050016 www.ijntr.org.

[50] Novakovic B M 2015 Int. J. New Techn. Res. 15 ID-IJNTR01050017 www.ijntr.org.

[51] Novakovic B M 2017 Int. J. New Techn. Res. 311 ID-IJNTR03110009 www.ijntr.org.

[52] Novakovic B M 2010 CP 1303 Computing Anticipatory Systems CASYS'09 Ninth Int. Conf. ad. D M Dubois - 2010 AIP 978-0-7354-0858-6/10/\$30.00

[53] Novakovic B M 2015 Int. J. New Techn. Res. 17 ID-IJNTR01070005 www.ijntr.org.

[54] Novakovic B M 2017 Int. J. New Techn. Res. 311 ID-IJNTR03110008 www.ijntr.org.

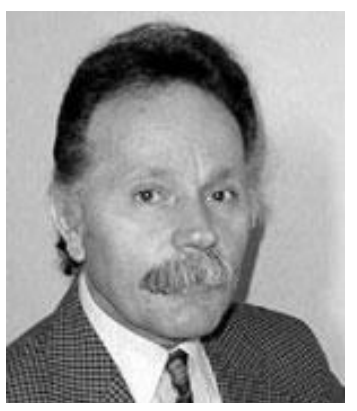

Branko Novakovic is a Professor emeritus at FSB - University of Zagreb, Croatia. Prof. Novakovic received his $\mathrm{PhD}$ from the University of Zagreb in 1978. His research of interest includes physics, control systems, robotics, neural networks, and fuzzy control. He is author of three books; Relativistic Alpha Field Theory (RAFT, e-book, 2016), Control Methods in Robotics, Flexible Manufacturing Systems and Processes (1990), Control Systems (1985) and the first co-author of a book Artificial Neural Networks (1998). He has published over 230 research papers in his research of interest. 